\title{
PLANEJAMENTO EXPERIMENTAL TIPO ESTRELA PARA ANÁLISE E DETERMINAÇÃO DA COMPOSIÇÃO DE MONOETILENOGLICOL, CLORETO DE SÓDIO E ÁGUA COM BASE EM MEDIDAS DE DENSIDADE E CONDUTIVIDADE ELÉTRICA A 298,15 K
}

\author{
A.R.C.SILVA ${ }^{1}$, D.J.SIVA ${ }^{1}$, L.S.PEREIRA ${ }^{2}$, J.F.NASCIMENTO² e O.CHIAVONE-FILHO ${ }^{1}$ \\ ${ }^{1}$ Universidade Federal do Rio Grande do Norte, Departamento de Engenharia Química \\ ${ }^{2}$ Petrobras/CENPES/PDDP/TPP \\ E-mail para contato: adriano.rafae195@ hotmail.com
}

\begin{abstract}
RESUMO - A unidade de regeneração do monoetilenoglicol (MEG) nas plataformas de produção de gás natural contém três etapas: pré-tratamento, recuperação e regeneração. $\mathrm{Na}$ etapa de recuperação são coletados dados de condutividade elétrica $(\mathrm{k})$ através da coluna de salmoura com sensores presente no separador flash e medidas de densidade $(\rho)$ são realizadas para o conhecimento da composição do sistema. Visando este processo, o objetivo do estudo é analisar e descrever a composição das fases do separador flash. Para isto, foi construído um planejamento experimental fatorial do tipo estrela $2^{2}$ com triplicata no ponto central e quatro pontos axiais tendo como variáveis independentes as concentrações mássicas de MEG e cloreto de sódio $(\mathrm{NaCl})$ e como variáveis dependentes a densidade e condutividade elétrica a 298,15 K. Foram realizadas análises estatísticas assim como correlações com os modelos matemáticos gerados no Statistica 13, além de testes com duas séries de dados de densidade e condutividade elétrica a $298,15 \mathrm{~K}$. Notou-se que a concentração de MEG é o fator de maior significância na densidade. Para a condutividade, a influência da concentração de MEG é levemente superior à de $\mathrm{NaCl}$ e apresentam comportamentos inversos. Para os modelos, os desvios estimados na correlação foram satisfatórios: $\Delta \rho=0,01 \%$, e $\Delta \mathrm{k}=0,12 \%$. Assim, o método demonstrou ser adequado para descrever a composição do processo de regeneração do MEG.
\end{abstract}

\section{INTRODUÇÃO}

O monoetilenoglicol (MEG) é utilizado na indústria do petróleo como inibidor de hidrato na produção de gás natural. Em condições favoráveis de temperatura e pressão pode ocorrer a formação de hidrato nas tubulações, provocando a interrupção da produção e prejuízos (Sandengen e Kaasa, 2006). Com a produção de petróleo e gás produz-se também água contendo íons em solução e em concentrações variadas dependendo de sua fonte e ambiente de deposição (Hunt, 1995). Os íons que estão em maiores concentrações nas águas de campo petrolífero são: sódio, cálcio, magnésio e cloreto. 
Devido à importância e a larga utilização do MEG na unidade de produção de gás natural existe uma unidade de regeneração do monoetilenoglicol que visa recuperar este produto de forma a reduzir os custos de produção e o impacto ambiental causado por ele. A unidade de regeneração do MEG contém três etapas: pré-tratamento, recuperação e regeneração. A primeira visa a remoção de hidrocarbonetos, a segunda a remoção de sais e a terceira a separação da água. A concentração de água no MEG que entra na segunda etapa (Separador Flash) é em torno de $42 \%(\mathrm{~m} / \mathrm{m})$. Abaixo deste separador existe uma coluna de salmoura com sensores de condutividade para controle do processo. $\mathrm{O}$ vapor que sai do separador, contendo água e MEG, é direcionado para uma coluna de destilação que finalmente regenera o MEG para reinjeção na cabeça do poço de produção com concentração em torno de $82 \%(\mathrm{~m} / \mathrm{m})$. Além da condutividade, medidas de densidade são realizadas a partir de amostras para o conhecimento da composição do sistema nas várias correntes do processo (Sandengen e Kaasa, 2006; Zemánková et al., 2013).

Largamente utilizado nas indústrias química e petroquímica, o processo de separação flash ou destilação flash pode ser definido como um processo de simples estágio que consiste na vaporização de uma fração definida da corrente material a ser separada, de tal forma que o vapor obtido neste processo é separado e condensado. Desse modo, o objetivo desse estudo é analisar e descrever o comportamento das fases do Separador Flash através do conhecimento das concentrações de MEG, cloreto de sódio $(\mathrm{NaCl})$ e água com base em medidas de densidade e condutividade a $298,15 \mathrm{~K}$.

\section{METODOLOGIA}

Visando investigar estatisticamente a influência das concentrações mássicas de MEG, $\mathrm{NaCl}$ e água na condutividade e densidade foi construído um planejamento experimental fatorial do tipo estrela $2^{2}$ (dois níveis e duas variáveis com triplicata no ponto central e quatro pontos axiais). Os experimentos ocorreram a 298,15 K e variaram as concentrações percentuais de $\mathrm{MEG}, \mathrm{NaCl}$ e, consequentemente, água. A Tabela 1 apresenta o intervalo de estudo das variáveis independentes que foram utilizadas para a construção do planejamento experimental.

Tabela 1 - Intervalos de estudos das variáveis independentes

\begin{tabular}{|c|c|c|c|c|c|}
\hline Variável & Nível inferior & Nível superior & Ponto central & $\alpha$ & $-\alpha$ \\
\hline$[\% \mathrm{MEG}](\%, \mathrm{~m} / \mathrm{m})$ & 20 & 80 & 50 & 92,3 & 7,7 \\
\hline$[\% \mathrm{NaCl}](\%, \mathrm{~m} / \mathrm{m})$ & 1 & 5 & 3 & 5,82 & 0,18 \\
\hline
\end{tabular}

Essas condições foram escolhidas por compreender os intervalos de concentrações encontrados na unidade de regeneração do MEG na plataforma de produção de gás natural.

Correlações com os modelos matemáticos gerados pela análise estatística foram realizadas, bem como testes de duas séries extensivas de dados de densidade e condutividade elétrica a 298,15 K. Os dados apresentam toda faixa de concentração de água e MEG, e também em termos de cloreto de sódio $(\mathrm{NaCl})$, compreendidos na faixa de concentração contemplada pelo planejamento experimental e de interesse da unidade de regeneração.

De posse das equações empíricas, foi realizado o cálculo inverso a partir da resolução do sistema de duas equações, isto é, a partir da densidade e condutividade é possível determinar a 
concentração do sistema em termos de $\mathrm{MEG}, \mathrm{NaCl}$ e água. Para tal, foi desenvolvido um código computacional no Scilab.

\section{RESULTADOS E DISCUSSÕES}

A Tabela 2 apresenta o resultado do planejamento experimental tipo estrela realizado para avaliar a influência das concentrações de monoetilenoglicol e $\mathrm{NaCl}$ nas medidas de densidade e condutividade a $298,15 \mathrm{~K}$.

Tabela 2 - Resultado do planejamento experimental a 298,15 K

\begin{tabular}{|c|c|c|c|}
\hline \multicolumn{2}{|c|}{ Variáveis estudadas } & \multicolumn{2}{|c|}{ Variáveis de resposta } \\
\hline$[\% \mathrm{NaCl}]$ & {$[\% \mathrm{MEG}]$} & Densidade $\mathrm{g} / \mathrm{cm}^{3}$ & Condutividade $(\mathrm{mS} / \mathrm{cm})$ \\
\hline-1 & -1 & 1,0301 & 7,631 \\
\hline 1 & -1 & 1,0590 & 51,423 \\
\hline-1 & 1 & 1,1018 & 2,139 \\
\hline 1 & 1 & 1,1302 & 8,721 \\
\hline 0 & 0 & 1,0837 & 16,080 \\
\hline 0 & 0 & 1,0840 & 16,068 \\
\hline 0 & 0 & 1,0841 & 15,984 \\
\hline$-1,41$ & 0 & 1,0638 & 1,194 \\
\hline 1,41 & 0 & 1,1049 & 27,465 \\
\hline 0 & $-1,41$ & 1,0281 & 42,780 \\
\hline 0 & 1,41 & 1,1257 & 3,830 \\
\hline
\end{tabular}

Após o tratamento estatístico dos dados utilizando o Statistica 13, obteve-se a Tabela 3, apresentando os efeitos bem como as interações entre as variáveis estudadas.

Tabela 3 - Efeitos e os valores de p obtidos para as variáveis do planejamento experimental

\begin{tabular}{|c|c|c|c|c|}
\hline & \multicolumn{2}{|c|}{ CONDUTIVIDADE } & \multicolumn{2}{c|}{ DENSIDADE } \\
\hline & Efeito & $\mathrm{P}$ & Efeito & $\mathrm{P}$ \\
\hline & 16,0491 & 0,000 & 1,083940 & 0,000 \\
\hline Média global & 21,8988 & 0,000 & 0,028881 & 0,000 \\
\hline $\mathrm{NaCl}(\mathrm{L})$ & $-2,4218$ & 0,302 & 0,000215 & 0,747 \\
\hline $\mathrm{NaCl}(\mathrm{Q})$ & $-25,8761$ & 0,000 & 0,070313 & 0,000 \\
\hline MEG (L) & 6,6071 & 0,026 & $-0,007254$ & 0,000 \\
\hline MEG (Q) & $-18,6462$ & 0,001 & $-0,000250$ & 0,751 \\
\hline
\end{tabular}

*(L) - Contribuição linear; (Q) - Contribuição quadrática

Os efeitos são tidos como significativos nos resultados das variáveis de resposta quando o valor de P é menor que 0,05 . A partir dessa análise é possível determinar quais variáveis apresentam um parâmetro significativo no modelo matemático que representa a variável dependente. Com base nos efeitos, o modelo polinomial proposto foi: $\mathrm{var}=\mathrm{a} 0+\mathrm{a} 1 .[\% \mathrm{NaCl}]+$ a2.[\% NaCl] $]^{2}+\mathrm{a} 3 .[\% \mathrm{MEG}]+\mathrm{a} 4 .[\% \mathrm{MEG}]^{2}+\mathrm{a} 5 .[\% \mathrm{NaCl}] .[\% \mathrm{MEG}]$. No modelo, a0, a1, a2, a3, a4 e a5 são parâmetros, no qual, a0 é a média global da variável de estudo e o restante são os efeitos causados pelas variáveis independentes e as interações entre elas.

No entanto, após analisar os resultados utilizando os modelos propostos para a densidade e condutividade desconsiderando os parâmetros não-significativos de acordo com os gráficos de Pareto, percebeu-se uma perda de precisão nas medidas tanto de condutividade quanto de densidade, evidenciada principalmente nos resultados do cálculo inverso combinando os dois 
modelos para encontrar as concentrações percentuais de $\mathrm{MEG}$ e $\mathrm{NaCl}$. A perda de precisão nos dados se deve ao fato de os resultados de densidade e condutividade serem muito sensíveis, qualquer pequena alteração nas condições de análise evidencia uma significativa diferença no resultado final. Sendo assim, adotou-se os modelos com todos os parâmetros:

$$
\begin{aligned}
& \text { Densidade }\left(\frac{g}{c^{3}}\right)=0,993539+0,007163 \cdot[\% N a C l]+0,0000269 \cdot[\% N a C l]^{2}+ \\
& \text { 0,001581. }[\% M E G]-0,00000403 \cdot[\% M E G]^{2}-0,00000208 \cdot[\% N a C l] \cdot[\% M E G] \\
& \text { Condutividade }\left(\frac{m s}{c m}\right): 4,3328138+15,0602953 \cdot[\% N a C l]-0,3027282 \cdot[\% N a C l]^{2}- \\
& \text { 0,332178. }[\% M E G]+0,0036706 \cdot[\% M E G]^{2}-0,1553847 \cdot[\% N a C l] \cdot[\% M E G]
\end{aligned}
$$

Os modelos obtiveram valores de coeficiente de correlação linear de 0,9998 e 0,9888 respectivamente, sinalizando ajustes razoáveis entre os dados experimentais e os calculados. A Figura 1 faz o confronto entre os dados observados e os preditos.

Figura 1 - Valores observado versus valores preditos pelos modelos polinomial para ajustes dos dados de densidade $\left(\mathrm{g} / \mathrm{cm}^{3}\right)$ com $\mathrm{R}^{2}=0,9998$ (a) e para os dados da condutividade

$(\mathrm{mS} / \mathrm{cm}) \operatorname{com} \mathrm{R}^{2}=0,9888(\mathrm{~b})$

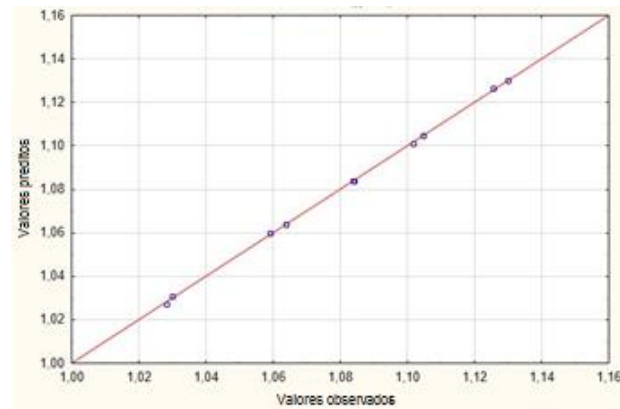

(a)

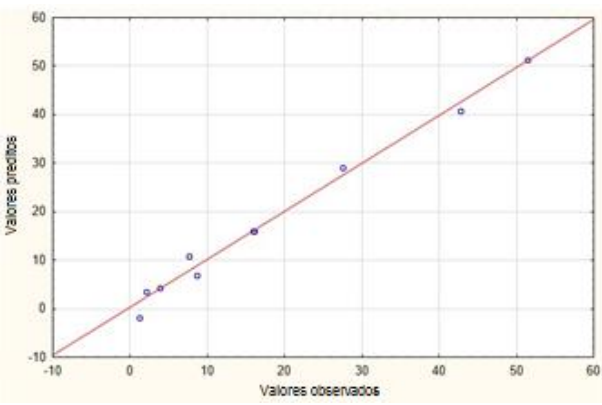

(b)

$\mathrm{Na}$ análise estatística realizada no software Statistica 13, os digramas de Pareto apresentaram os efeitos causados individualmente pelas variáveis de estudo nas variáveis de resposta, bem como suas interações. Observou-se que algumas variáveis apresentaram valores absolutos positivos e outras valores negativos, este sinal indica se a influência no resultado final da variável de resposta será de forma a aumenta-la (quando for positiva) ou diminui-la (quando for negativa). Notou-se que para a densidade, discrepantemente, a concentração de MEG é o fator de maior significância, exercendo uma influência quase que três vezes maior que a concentração de sal. Para a condutividade, a influência da concentração de $\mathrm{MEG}$ e $\mathrm{NaCl}$ no meio são semelhantes, mas com formas de contribuição contrárias, já que apresenta, respectivamente, sinal negativo e positivo, como esperado.

Analisou-se ainda os resíduos e estes comprovaram a validade dos modelos e a significância da regressão, uma vez que os pontos flutuaram e tiveram sua distribuição aleatória em torno de zero. Observou-se que os dados de densidade apresentam erros muito pequenos, com variação apenas na terceira casa decimal após a vírgula, isso ocorre devido a precisão dos instrumentos utilizados e da baixa variação dos valores de densidade para as diferentes concentrações de $\mathrm{MEG}$ e $\mathrm{NaCl}$, tornando a predição feita pelo modelo mais eficiente. Porém, os resíduos dos valores de condutividade apresentaram um desvio mais significativo, isso 
ocorre por erros inerente ao instrumento usado, mesmo utilizando duas células diferentes para aumentar a precisão: a de $\mathrm{k}=0,1 \mathrm{~cm}^{-1}$ para concentrações com até $1 \%$ de $\mathrm{NaCl}$ e a com $\mathrm{k}=10$ $\mathrm{cm}^{-1}$ para as demais. Além disto, o modelo abrange valores de condutividades significativamente baixas e altas, dificultando a precisão do modelo em todos os pontos.

Também foram analisadas as superfícies de resposta, disposta na Figura 3. Observando a superfície de resposta referente a densidade nota-se que a medida que aumenta a concentração de MEG em solução a densidade tende a aumentar devido a sua viscosidade elevada. Nota-se ainda que a concentração de $\mathrm{NaCl}$ na solução tem pequena influência no resultado final. Analisando a condutividade, pode-se observar que elevadas concentrações de $\mathrm{NaCl}$ eleva a condutividade, fato esperado devido a maior disponibilidade de íons livres. Ao contrário do que se esperava, o fator de maior influência é a concentração de MEG, no entanto, com comportamento inverso, ou seja, a medida que se eleva a fração de MEG diminui a condutividade final, pois o MEG diminui a mobilidade dos íons no meio.

Figura 3 - Superfície de resposta da densidade (a) e condutividade (b) em função da concentração de MEG e $\mathrm{NaCl}$

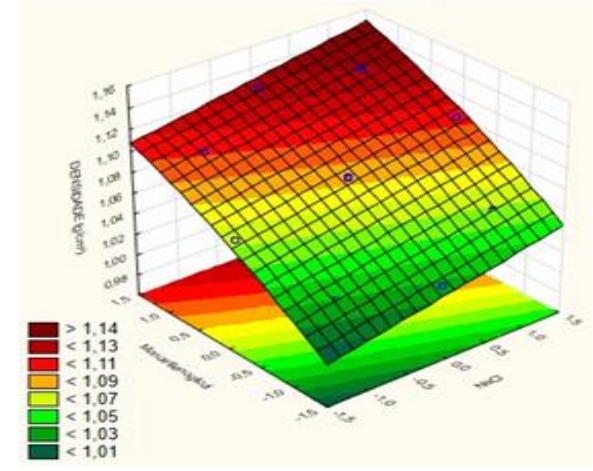

(a)

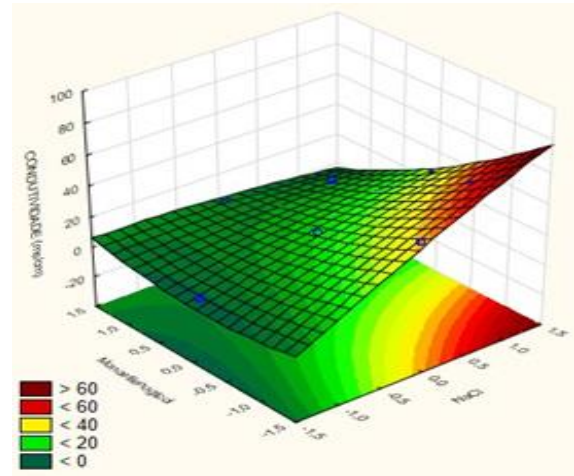

(b)

Correlações com as modelos matemáticos gerados pela análise estatística foram realizadas das duas séries extensivas de dados de densidade e condutividade elétrica a 298,15 K (dados do grupo de pesquisa FOTEQ da UFRN). Foram analisados 31 dados experimentais de densidade e 17 de condutividade elétrica. A correlação para a densidade apresentou $\mathrm{R}^{2}=$ 0,9990 e a de condutividade $\mathrm{R}^{2}=0,9922$, evidenciando que os modelos descrevem bem os dados experimentais garantindo confiabilidade nos resultados. O intervalo de confiança das correlações é de 0,95 .

A partir da correlação entre os dados calculados e os experimentais de condutividade e densidade, percebe-se, no entanto, que para dados com baixas condutividades o modelo apresenta maiores erros, apresentando pontos fora do intervalo de confiança, porém à medida que os resultados da condutividade são maiores, estes ganham maior precisão na predição do modelo. Esta imprecisão pode estar relacionada a imprecisão de medidas durante os procedimentos operacionais.

Um algoritmo no Scilab 5.4.1 foi desenvolvido para fazer o cálculo inverso a partir da resolução do sistema de duas equações, ou seja, a partir dos modelos de densidade e condutividade foram determinados a [\%MEG], [\% NaCl] e, por diferença, $\left[\% \mathrm{H}_{2} \mathrm{O}\right]$. A partir dos resultados calculados foram realizadas correlações com os dados experimentais neste 
planejamento experimental, conforme ilustrado na Figura 5. O intervalo de confiança das correlações é de 0,95. Para [\%MEG] o $\mathrm{R}^{2}=0,9903$ e para $[\% \mathrm{NaCl}]$ o $\mathrm{R}^{2}=0,9660$.

Como já analisado, a estimativa da [\%MEG] é majoritariamente determinada pela densidade, o modelo mais ajustado e com menor erro. Entretanto, a estimação da [\% $\mathrm{NaCl}]$ depende fundamentalmente da condutividade e este possui imprecisões no modelo inerentes ao procedimento operacional, como instrumentação por exemplo.

Figura 5 - Correlação da [\%MEG] realizada pelo Scilab pela [\%MEG] experimental (a) e correlação da $[\% \mathrm{NaCl}]$ realizada pelo Scilab pela $[\% \mathrm{NaCl}]$ experimental (b)

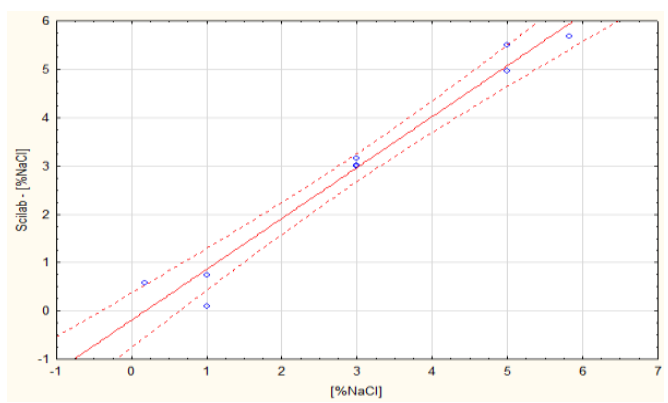

(a)

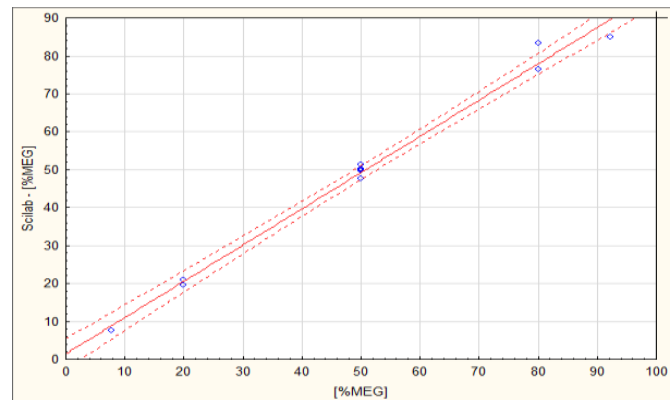

(b)

\section{CONCLUSÕES}

Através da análise estatística nota-se que a concentração de MEG é o fator de maior significância na densidade, exercendo uma influência quase que três vezes maior que a concentração de sal. Para a condutividade, a influência da concentração de MEG é levemente superior a de $\mathrm{NaCl}$ e apresentam comportamentos inversos, à medida que se eleva a quantidade de MEG diminui a condutividade, enquanto que a medida que se aumenta a quantidade de $\mathrm{NaCl}$ aumenta a condutividade. Para os modelos, os desvios estimados na correlação foram satisfatórios, dada a sensibilidade dos dados e proximidade da incerteza experimental, ou seja, $\Delta \rho=0,01 \%$, ou $0,0001 \mathrm{~g} / \mathrm{cm} 3$, e $\Delta \mathrm{k}=0,12 \%$, ou $0,0189 \mathrm{mS} / \mathrm{cm}$. Com o algoritmo desenvolvido foi possível recalcular a série de dados do planejamento experimental e os erros absolutos em fração mássica e relativos percentual foram, respectivamente, de 0,1152 ou $0,32 \%$ para $\Delta[\% \mathrm{MEG}]$ e de 0,0039 ou $0,13 \%$ para $\Delta[\% \mathrm{NaCl}]$. Tendo em vista essa satisfatória reprodutibilidade o método demonstrou ser adequado para descrever a composição da fase líquida do processo de regeneração do MEG a partir das informações de densidade e condutividade elétrica.

\section{REFERÊNCIAS}

HUNT, J.M. 1995. Petroleum geochemistry and geology. Ed. W.H. Freeman and Company. 2nd. Ed., New York, 743p.

SANDENGEN, K., KAASA, B., Estimation of MEG content in water + $\mathrm{MEG}+\mathrm{NaCl}+$ NaHCO3 solutions, J. Chem. Eng. Data, 51, 443-447, 2006.

ZEMÁNKOVÁ, K., TRONCOSO J., ROMANÍ, L. Excess volumes and excess heat capacities for alkanediol + water systems in the temperature interval (283.15-313.15) K. Fluid Phase Equilibria 356, 1-10, 2013. 